\title{
An Empirical Propagation Model for Corridors in Office Buildings
}

\author{
Paweł Kosz
}

\begin{abstract}
This paper presents an empirical propagation path loss model for corridors in office buildings. The proposed model estimates changeable character of radio signal attenuation, based on a special approach as a combination of the simple free-space model with the author's model. The measurement stand and measurement scenario are described. The propagation path loss research have been made in corridor for different frequencies in range $30 \mathrm{MHz}$ to $290 \mathrm{MHz}$. A significant number of measurement results were allowed an analysis of the radio wave propagation conditions in the environment. In general, the propagation path loss increases for each measurement frequencies with length of propagation route. Based on measurement data, the new empirical propagation path loss model was developed. For this purpose, the regression analysis was made. The novelty of this model is that it could be used for estimate propagation path loss in measured environment for different radio wave frequencies. At the end, in order to justification the practical usefulness of described method for estimate a radio wave attenuation, the statistical evaluation was made. Thus, the results of the statistical analysis (ME, SEE and $R^{2}$ values) are satisfactory for each measured radio wave frequency.
\end{abstract}

Keywords-wireless systems, radio wave propagation, propagation model, propagation path loss, indoor environment, test stand

\section{INTRODUCTION}

$\mathbf{N}$ OWADAYS, the radio communication networks are key factor in process of telecommunication infrastructure development. Due to realisation of wireless data transmission using radio waves propagation, we could ensure the access to telecommunication services for a large number of users on the specified area without installation of permanent wire system. This allows for an unlimited extension and reconfiguration of the system structure, but on the other hand it requires a special approach to the process of planning a radio communication networks. It should be noted that for designing that networks properly, the propagation conditions of a transmission channel has to be known, because this properties have a crucial influence on quality of radio transmission parameters [1].

As it is known, one of the most important phenomenon in radio channels is the radio wave attenuation (so-called propagation path loss [2]). It is a parameter, that determine the level of reduction in power density of an electromagnetic wave. The radio wave attenuation is determined by many variable factors such as: type of environment (outdoor or indoor), length of the propagation distance, environment geometry, atmospheric conditions, so the estimation process of

P. Kosz is with the Department of Radiocommunication Systems and Networks, Faculty of Electronics, Telecommunications and Informatics, Gdansk University of Technology, Gabriela Narutowicza St. 11/12, 80-233 Gdansk, Poland (e-mail: pawel.kosz@eti.pg.gda.pl). propagation path loss is very difficult and complicated issue. Knowledge of the transmission attenuation is necessary to meet energy requirements in radio links designing [3]. Relevant to measuring research in connection with knowledge of propagation condition and statistics methods, it is possible to create new empirical propagation models to estimate the radio wave attenuation with satisfactory accuracy [4].

The radio wave attenuation issue in indoor environments, in particular for corridors in office buildings is widely discussed in literature [5]-[7]. Predominantly, the research area only focuses on Radiocommunication systems, that work in the license-free band [8]-[10]. It is known that, in the described environment, may need to be used the Radiocommunication systems, that worked in other useful radio wave frequencies. For instance, the mobile dispatch systems, which the main purpose is works in outdoor environments, in some situations, it is necessary to work in harsh, indoor environment [11][12]. Furthermore, a detailed study of the technical literature reveals, that some works are generally inconsistent and ambiguous, because they don't describe precisely the measuring procedure and the obtained results of analysis are based on relatively small number of data sets of power density of an electromagnetic wave [13][14].

Taking into consideration the above, in this article the procedure of measurement research and analysis of the radio wave propagation conditions in selected corridor in office buildings environment for different frequencies in range $30 \mathrm{MHz}$ to $290 \mathrm{MHz}$ was described. Choice for that frequency range was related to mobile dispatch systems, which in some situations may need to be worked in harsh, indoor environment. Based on obtained results, the empirical propagation model for estimation the radio wave attenuation in this case was developed. The measurements were part of the scientific-research project, realised in the Development Projects Laboratory in the Department of Radiocommunication Systems and Networks, Gdansk University of Technology, concerning monitoring people in harsh, indoor environment [15].

\section{MEASUREMENTS IN REAL ENVIRONMENT}

Corridors in office buildings have the characteristics, which determine the radio wave propagation conditions, what has a significant impact on the estimation process of path loss in this environment [6]. The radio wave propagation conditions in corridor in office buildings environment mainly depend on his construction, namely: cross-section dimensions, length of corridor and materials from which the walls had been made [5][7]. The most common realisation of described environment is a long rectangular corridor, where the propagation is similar to a waveguide [16][17]. Another 
important feature of the investigated environment is the occurrence of specific additional elements, that have impact on the radio wave propagation, such as: staircases, lift shafts, crossed corridor, suspended ceilings [10]. It needs to be highlighted that the presence of people in research environment has a significant influence of the radio signal propagation along a line-of-sight path [18].

\section{A. Test Bed for Propagation Path Loss Measurements}

In order to carry out of the propagation path loss measurements in real condition, the relevant measurement stand is required. It was realised in the Department of Radiocommunication Systems and Networks, Gdansk University of Technology. The designed measurement unit includes devices, which enable implementation of transmitting and receiving section of radio communication system.

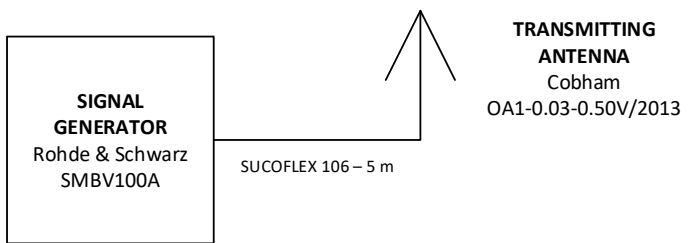

Fig. 1. Block diagram of transmitting section of measurement stand.

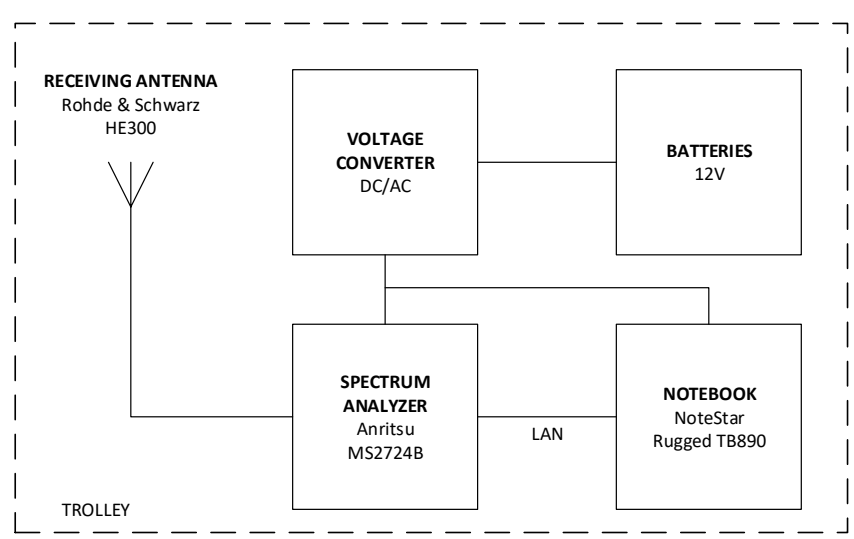

Fig. 2. Block diagram of receiving section of measurement stand.
In Fig. 1 a block diagram of equipment set of transmitting section used in propagation measurements is presented. The transmitting section of measurement stand consists of transmitting antenna and signal generator. The measurement devices were joined together via cable with a length of $5 \mathrm{~m}$. The frequency range of used generator is $9 \mathrm{kHz}-6 \mathrm{GHz}$. This device allows generation of radio signal compatible with many wireless standards e.g. GSM/EDGE, 3GPP FDD, IEEE 802.11a/b/g/n, WiMAX IEEE 802.16, DVB-T, LTE [19]. In transmitting section, the high performance, broadband whip antenna with a length of $1 \mathrm{~m}$ was used. This antenna is designed for use over the $30 \mathrm{MHz}$ to $512 \mathrm{MHz}$ frequency band. Presented antenna and its parameters allowed to use it in realized measurement stand [20].

In Fig. 2 a block of diagram of equipment set of receiving section is presented. In the receiving section a spectrum analyzer, receiving antenna and notebook with dedicated special software were used. The active directional antenna was connected with spectrum analyzer by cable. The three exchangeable antenna modules supplied with the antenna cover the $20 \mathrm{MHz}$ to $7.5 \mathrm{GHz}$ frequency range [21]. So, it was possible to use this device in described measurements. The used signal spectrum analyzer working as a sensitive received signal power meter and also as a data source for notebook [22], where special software for control the analyzer and save measurement data was installed. All the receiver section components were battery powered via DC/AC voltage converter. Additionally, in order to do the measurements in real conditions, all equipment from receiving section were placed on the trolley. In this way, it was possible to place easily the receiving section in many different positions in the area of corridor in office building. This selected equipment allowed to make measurements of radio wave path loss in real conditions according to the proposed scenario.

\section{B. Measurement Scenario for Propagation Environment}

To perform the measurement campaign in real research environment, the corridor in building A of Faculty of Electronics, Telecommunications and Informatics, Gdansk University of Technology was selected. Due to its characteristic simple construction, the selected corridor

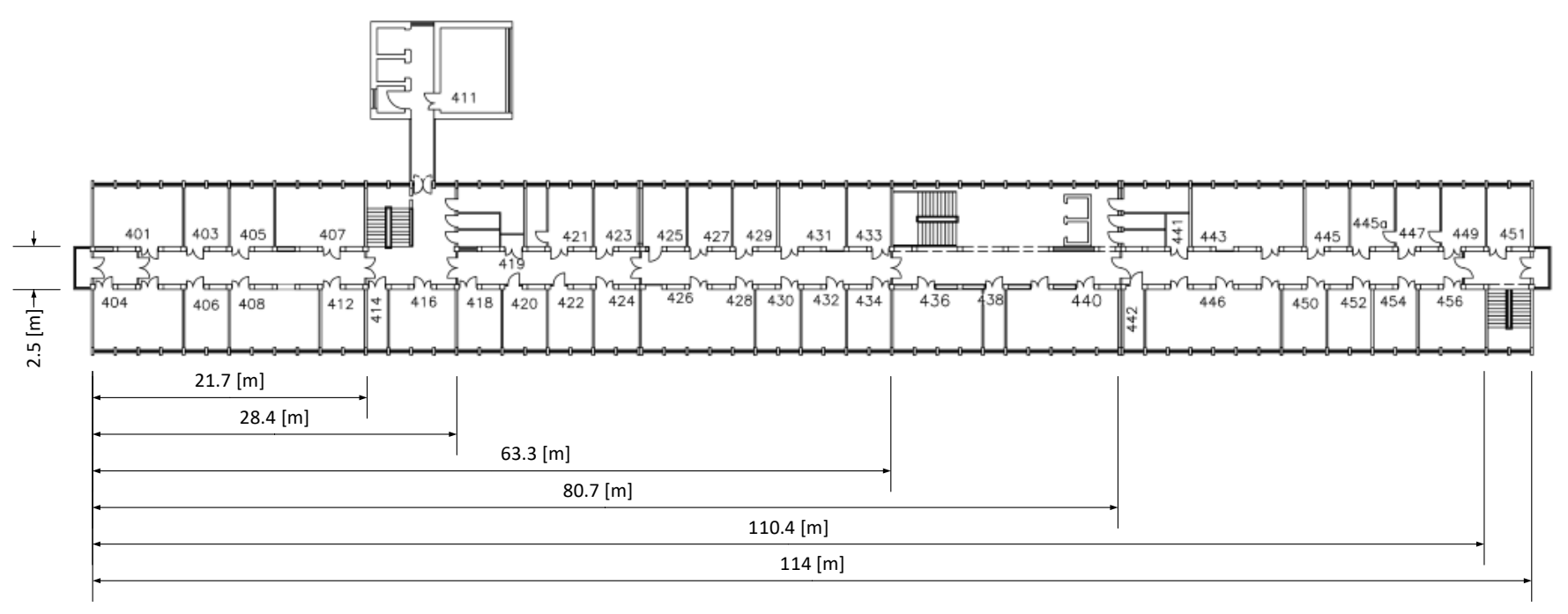

Fig. 3. The plan view of corridor on the 4th floor in the building of Faculty of Electronics, Telecommunications and Informatics. 
resembles a typical corridor that can be found in many office buildings. The propagation path loss measurements have been made in corridor on the $4^{\text {th }}$ floor for different frequencies in range $30 \mathrm{MHz}$ to $290 \mathrm{MHz}$. The measurement campaign was made in working hours, which means that people (employees and students) sometimes were walking up and down on the corridor, between transmitting and receiving antennas, so this allowed ensure maximally real radio wave propagation conditions.

The plan view of selected corridor, which the propagation path loss measurements were made, is shown in Fig. 3. Whereas in Fig. 4 the cross-section of corridor on the $4^{\text {th }}$ floor is presented. The length of the selected corridor is $114 \mathrm{~m}$, the width is $2.5 \mathrm{~m}$, the ceiling height is $3 \mathrm{~m}$ but taking into account suspended ceiling, the height is $2.8 \mathrm{~m}$. The thickness of each ceiling between floors is $0.5 \mathrm{~m}$. Additionally, wall thickness was also measured, this value approximates around $0.4 \mathrm{~m}$. In research environment, there are three staircases, at a distance of $22 \mathrm{~m}, 64 \mathrm{~m}$ and $110 \mathrm{~m}$ from the beginning of corridor.

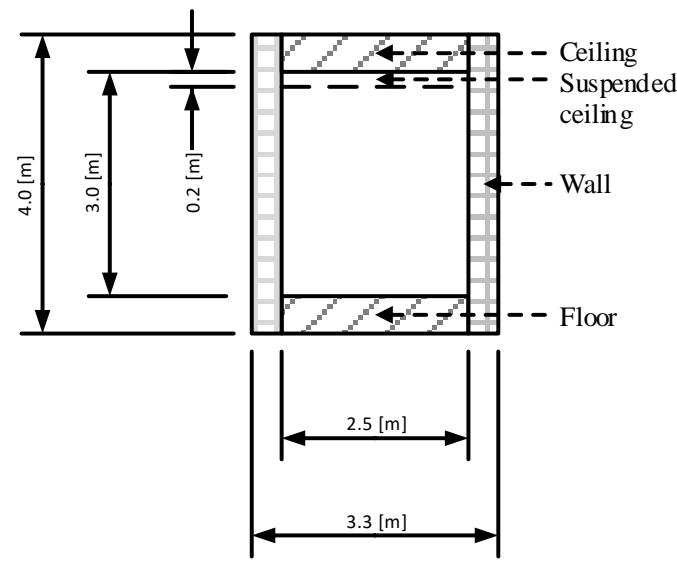

Fig. 4. The cross-section of corridor on the 4th floor in the building of Faculty of Electronics, Telecommunications and Informatics.

The transmitting antenna was placed on the axis of the corridor, at a distance of 1 meter from the beginning. The height of the middle part of antenna was $1.5 \mathrm{~m}$. The signal generator was mounted in office room, which was near to the transmitting antenna. The receiving antenna was mounted on tripod on the trolley, whereby the height of the middle part of this antenna was also $1.5 \mathrm{~m}$. During measurement, the receiving antenna always was directed to the source of the signal.

Namely, the propagation path loss measurements have been made in function of length of the propagation route at 9 different frequencies of radio signal: 30, 50, 70, 90, 120, 150, 170, 230 and $290 \mathrm{MHz}$. For the first seven frequencies, the measurement points were spaced with the $1 \mathrm{~m}$ distance apart, whereas for the last the highest two frequencies with the $0.5 \mathrm{~m}$. For each determined point, 50 instantaneous measurement values of power density of an electromagnetic wave were recorded. So, during measurement campaign in corridor, the 59500 data sets of power density of an electromagnetic wave were collected, which means about 7000 data for each analyzed frequency. This allowed to prepare a thorough analysis of propagation path loss for corridors in office buildings.

\section{AnAlysis of Propagation PATH Loss Results}

A significant number of measurement results allowed to define propagation path loss in discussed environment. As it is known, based on the instantaneous measurement values of power $P_{M R}[\mathrm{dBm}]$ available at the input of the receiving section of measurement stand, and also on the stored calibration data such as equivalent isotropic radiated power $E I R P[\mathrm{dBm}]$ and the absolute isotropic gain of the receiving antenna $G_{R}[\mathrm{dBi}]$, the instantaneous transmissions propagation loss values in real condition are determined by [3][23]:

$$
L_{\text {inst }}[d B]=E I R P[d B m]-P_{M R}[d B m]+G_{R}[d B i] .
$$

Then the results were analyzed as a propagation path loss characteristics in function of distance for each measurement frequency. On the presented graphs (Fig. 5), the instantaneous transmissions propagation loss values $L_{i n s t}$ and calculate median propagation loss values $L_{\text {med }}$ of each measurement point were highlighted. Additionally, for comparison, on the each graph the characteristic of free-space basic transmission loss $L_{f s}$ was drawn. In Fig. 5 a selected propagation path loss characteristics in function of distance is presented.
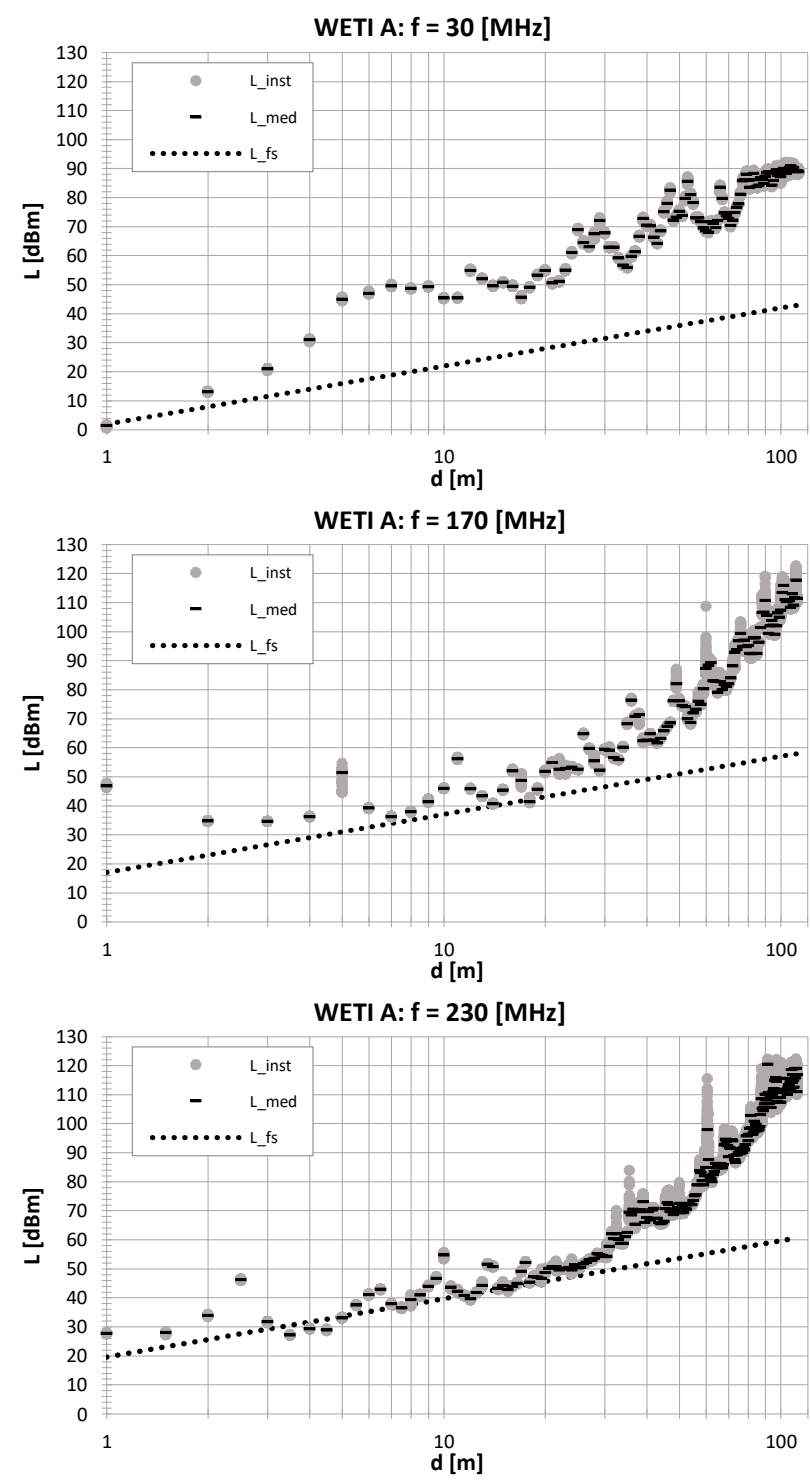

Fig. 5. Selected propagation path loss characteristics in function of distance. 
In frequency range $30-70 \mathrm{MHz}$ the determined propagation attenuation is significantly greater than in free-space condition case for whole distance between transmitting and receiving section. For this frequencies, the increment of propagation loss with the distance is bigger than in free-space case. Another observation is that for frequency $70 \mathrm{MHz}$ and higher, can be distinguished two, clearly seen areas with different inclination characteristics of propagation loss. For little distance from transmitting antenna there is the near region where the propagation loss is smaller and it is comparable with the path loss for free space. For long distance from transmitting antenna there is the far region where the propagation loss is larger, where the destructive interference dominates. Additionally, for this range of frequencies and for distance between $20-30$ and $60-80$ meters, may be notice that there are specific, larger values of radio signal attenuation. This is because, in this region of corridor is staircase.

\section{The Proposal Propagation Path Loss Model For MEASUREMENT ENVIRONMENT}

In order to develop new empirical propagation path loss model the regression analysis was made [1][5]. It is obvious, that the regression analysis is a statistical process for estimating the relationships among variables, which rely on selecting a specific linear regression function, estimating parameters and statistical evaluating of measurement results. Preliminary analysis of the propagation path loss (see chapter III) has shown that in described environment occur two propagation regions with different inclination characteristics of propagation attenuation. For short distance, there is a near region where the propagation path loss values are similar to the propagation path loss values calculated by free-space basic transmission model [18][24]. Whereas, for longer distance, there is a far region where the propagation path loss values had the exponential growth with function of distance. Therefore, the proposed propagation path loss model $L_{e s t}$ is a special approach, which based on combine with the simple free-space basic model $L_{f s}$ and the authors additional model $L_{a d d}$ to estimate changeable character of radio signal attenuation in presented environment. This proposed model, as a regression function which representing the relationship between dependent variable and independent predictors [25], and also as a definition about modeled phenomenon of propagation path loss for corridors in office buildings is given by:

$$
L_{e s t}[d B]=L_{f s}[d B]+L_{a d d}[d B] .
$$

In order to predict and estimate the propagation loss in freespace and also in various spaces with the first Fresnel zone clearance, a well-known basic model was used:

$$
L_{f s}[d B]=20 \lg \left(\frac{4 \pi d}{\lambda}\right),
$$

where $d$ is a distance between transmitting and receiving antenna, and $\lambda$ is a wavelength.

For estimate the changeable character of radio signal propagation path loss in presented environment, based on measuring empirical research results and also on observing the exponential growth of radio signal attenuations, the following instance of the function was proposed:

$$
L_{a d d}[d B]=\frac{1}{p_{1}(f)}[\lg (d)]^{p_{2}(f)},
$$

where $p_{1}(f), p_{2}(f)$ are searched unknown functions of - $f$. Process of estimation unknown function consists of determine this by least squares method [25]. Namely, the determined regression parameters had allowed for estimation of propagation path loss level with satisfying accuracy only for own measurement frequency. Indeed, during the process of develop a new mathematician model, the author is trying to create a function, that will be more universal and easy to use not for constant values but for wider range of parameters. For create a universal model, the founded parameters have been tested for changes in the frequency domain. This led to finding the dependence, which gave satisfactory coefficient of determination value. For both sets of searched functions, the similar relations between them the next values were observed. This method enabled to determine the more universal propagation model, which can estimates attenuation for any frequency from determined ranges. Next, using a research method based on curvilinear dependences, the equations describing changes in the frequency domain for parameters $p_{1}$ and $p_{2}$ were elaborated as:

$$
\begin{aligned}
& p_{1}(f)=\left\{\begin{array}{cc}
7.65[\lg (f)]^{2}-24.25 \lg (f)+19.17 & \text { a }) \\
-830.43[\lg (f)]^{2}+3340.2 \lg (f)-3335.5 & b) \\
-35.94[\lg (f)]^{2}+166.17 \lg (f)-191.05 & c)
\end{array}\right. \\
& p_{2}(f)=\left\{\begin{array}{cc}
59.89[\lg (f)]^{2}-187.43 \lg (f)+147.14 & a) \\
-207.1[\lg (f)]^{2}+831.15 \lg (f)-823.28 & b) \\
-106.29[\lg (f)]^{2}+488.13 \lg (f)-554.59 & c)
\end{array}\right.
\end{aligned}
$$

where equations $a$ are for the frequencies range <30,90) [MHz], equations $b$ are for $<90,170)$ [MHz] and equations $c$ are for $\langle 170,290\rangle$ [MHz].

Such prepared model could be used for estimate propagation path loss in measured environment for different radio wave frequencies (from $30-290 \mathrm{MHz}$ range). Whereby, the proposed idea is very useful for person, who designed a new radio communication system in harsh indoor environments which are corridors in office buildings. This method is easy to use and universal, mostly during first stage of wireless system design. In Fig. 6 the selected propagation path loss characteristics in function of a distance is presented, both for measured instantaneous propagation loss values $L_{i n s t}$ and also for estimated values using the proposed model $L_{e s t}$.

In order to justification the practical usefulness of described method for estimate a radio wave attenuation, the statistical evaluation for newly proposed empirical propagation model was made. The subject of evaluation was comparison the estimated values with the measured values of propagation attenuation. At first, the Mean Error $(M E)$, as the average difference between measured and estimated values, was determined. The $M E$ measures the average error, that could be expected in predicting behaviour of the model in further research and when values of error are closer to zero then better. The mean error is given by:

$$
M E[d B]=\frac{1}{N} \sum_{i=1}^{N}\left(L_{m e d}[d B]-L_{e s t}[d B]\right),
$$

where $L_{m e d}$ is the measured median propagation loss, $L_{e s t}$ is the estimated attenuation value calculated using the proposed model, and $N$ is a number of measurements for given scenario. 

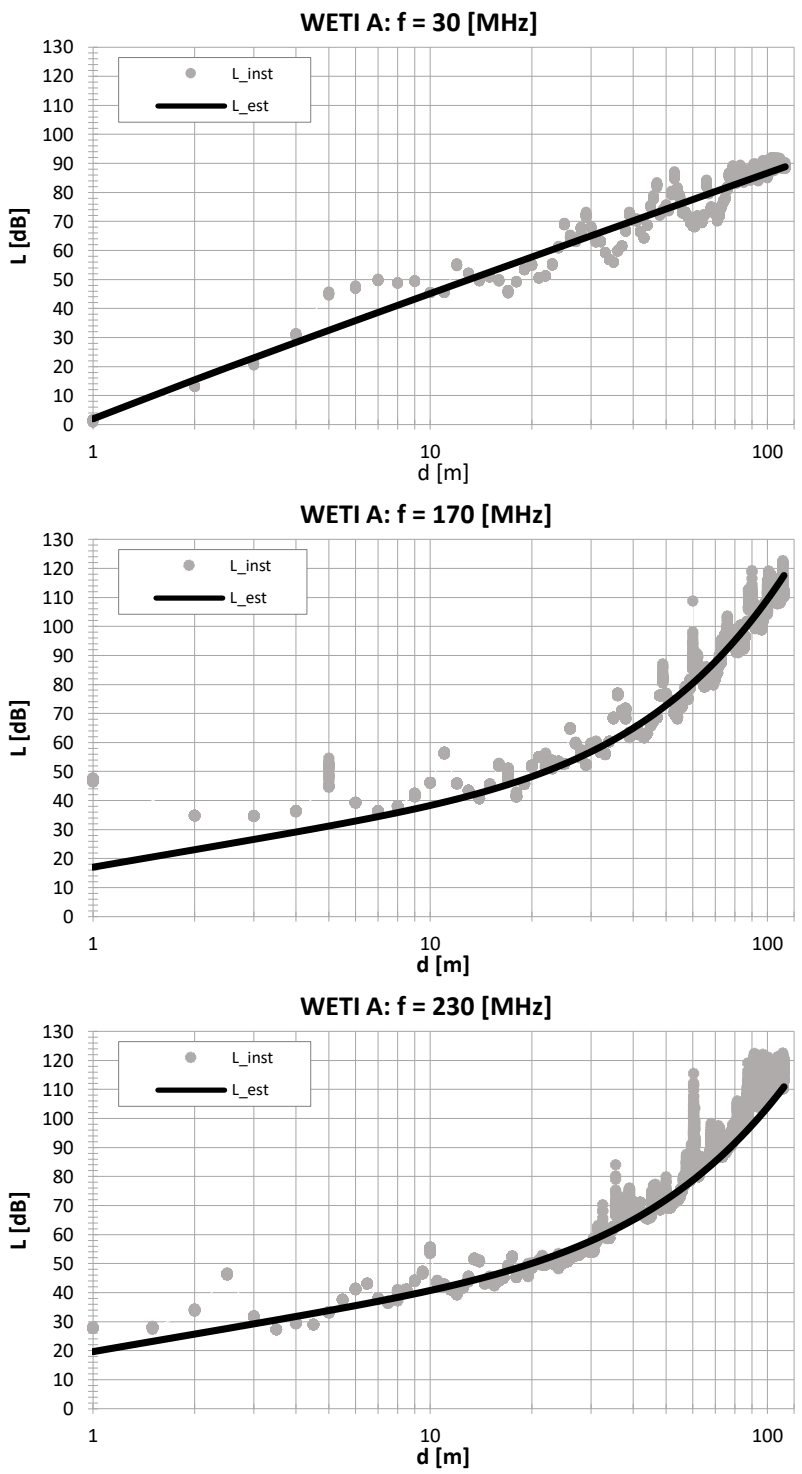

Fig. 6. Selected propagation path loss characteristics in function of distance, both for measured values and for estimated values.

Next, the Standard Error of Estimate (SEE), as the square root of the mean of the squares of the difference between measured and predicted values, was calculated. This measure determines the accuracy of predictions based on specified regression equation. Based on [12], the limited and acceptable values of this error are under $8[\mathrm{~dB}]$. This error may be presented as:

$$
S E E[d B]=\sqrt{\frac{1}{N-1} \sum_{i=1}^{N}\left(L_{m e d}[d B]-L_{e s t}[d B]\right)^{2}} .
$$

Additionally, in order to make the statistical analysis more reliable and define the accuracy of predictions, the coefficient of determination $\left(R^{2}\right)$ was calculated for each frequency. In statistics, this parameter indicates the ratio of the variance in a dependent variable that is predictable from an independent variable. The coefficient of determination ranges from 0 to 1 . That is obvious, if this parameter is closer to unity, the empirical model explains better the analysed phenomenon. In Table 1 the obtained results of usefulness for described method for estimate a radio wave attenuation are presented.
The results of the statistical analysis of newly proposed empirical propagation model are satisfactory. The mean error for most tested frequencies oscillates within the range of about -1 to 1 . This shows that, the proposed model has good accuracy and the process of analysis was performed correctly. The exception is the result of frequency $230 \mathrm{MHz}$, where the mean error is above $3[\mathrm{~dB}]$, due to the fact that for this frequency the single, strong disturbance of temporary measurement values of power density of an electromagnetic wave were recorded. The reason for this was the increased presence of people between transmitting and receiving antennas in this time. For all analysis frequencies, the very good results for the standard error of estimate were obtained. It shows also, that in this case the proposed mathematical model was good fitted with the empirical data. All the results of this measure are below the maximum acceptable value of this error. Whereas, the obtained coefficients of determination for whole range of frequencies is at least 0.91 and most of the cases is the value above 0.94 . This mean that, $94 \%$ of variability of propagation attenuation has been explained by the model. Thus, the proposed empirical propagation model quite good defined the variability of attenuation in investigated environment [1][5].

TABLE I

THE Summary Result of THE STATISTICAL ANALYSIS OF RESEARCH

\begin{tabular}{cccccc}
\hline \hline $\mathrm{F}$ & $\mathrm{p} 1$ & $\mathrm{p} 2$ & $\begin{array}{c}\mathrm{ME} \\
{[\mathrm{dB}]}\end{array}$ & $\begin{array}{c}\mathrm{SEE} \\
{[\mathrm{dB}]}\end{array}$ & $\mathrm{R}^{2}$ \\
30 & 0.04 & 0.96 & -0.34 & 5.17 & 0.91 \\
50 & 0.05 & 1.57 & -0.72 & 6.9 & 0.91 \\
70 & 0.47 & 5.2 & -0.99 & 4.52 & 0.98 \\
90 & 20.6 & 10.06 & -0.85 & 4.65 & 0.98 \\
120 & 19.44 & 9.54 & -0.21 & 5.04 & 0.94 \\
150 & 0.68 & 5.204 & 0.51 & 3.91 & 0.97 \\
170 & 0.79 & 5.376 & 1.11 & 6.04 & 0.93 \\
230 & 0.93 & 5.38 & 3.17 & 5.71 & 0.94 \\
290 & 0.2 & 2.901 & -1.24 & 4.74 & 0.94 \\
\hline For all measurement data & & 0.04 & 5.18 & 0.94 \\
\hline \hline
\end{tabular}

\section{CONCLUSIONS}

To sum up, in this paper, the procedure of measurement research and analysis of the radio wave propagation conditions in selected corridor in office buildings environment were described. To perform the measurement campaign in real environment, the relevant measurement stand was realised. After measurement in corridor in building A of Faculty of Electronics, Telecommunications and Informatics, Gdansk University of Technology, while the 59500 data sets of power density of an electromagnetic wave have been collected, the path loss characteristics in function of along the route were expunged. This allowed to prepare a thorough analysis of propagation path loss conditions for corridors in office buildings. The analysis showed that, the obtained values of propagation attenuation are consistent with the theoretical paradigms. For each measured frequency demonstrated that, 
with increasing distance between antennas, the propagation path loss is also increasing. Therefore, the length of the propagation route and the radio wave frequency are parameters that should be directly related with the propagation attenuation in office corridor or in any other researched propagation environment [4][16]. It is known that, the important influence on the radio wave propagation conditions in corridor in office environment had also its construction, namely: cross-section dimensions. Therefore, future work should be planed for corridors with other different dimensions.

Finally, in the last stage of work, based on recorded measurement data, the new empirical propagation path loss model was developed. It is a special approach, which based on combine with the simple free-space basic model and the author's model to estimate changeable character of radio signal attenuation in presented environment. The proposed model is universal and it can estimate radio wave attenuation for any frequency from determined ranges in described harsh indoor environment. Whereas, the results of the statistical analysis of newly proposed empirical propagation model are satisfactory for each measured radio wave frequency. Thus, the proposed empirical propagation model really well describes the variability of attenuation in investigated environment.

\section{REFERENCES}

[1] L. Barclay, "Propagation of Radio Waves", London: The Institution of Engineering and Technology, 2012.

[2] ITU-R V.573-5, "Radiocommunication Vocabulary", 2007.

[3] ITU-R P.341-5, "The Concept of Transmission Loss for Radio Links", 1999.

[4] W. C. Y. Lee, "Mobile Communications Design Fundamentals - Second Edition", New York: John Wiley \& Sons, 1993.

[5] M. Tolstrup, "Indoor Radio Planning - A Practical Guide for 2G, 3G and 4G", Chichester: John Wiley \& Sons, 2015.

[6] H. Hashemi, "The Indoor Radio Propagation Channel", Proceeding of the IEEE, vol. 81, pp. 943-968, 1993.

[7] J. Cheung, J. Sau, R. Murch, "A New Empirical Model for Indoor Propagation Prediction", IEEE Transactions on Vehicular Technology, vol. 47, pp. 996-1001, 1998.
[8] J. Medbo, J. Berg, "Simple and Accurate Path Loss Modeling at $5 \mathrm{GHz}$ in Indoor Environments with Corridors, IEEE Vehicular Technology Conference, pp. 30-36, 2000.

[9] X. Zhao, S. Geng, B. Moussa Coulibaly, "Path-Loss Model Including LOS-NLOS Transition Regions for Indoor Corridors at $5 \mathrm{GHz}$ ", IEEE Antennas and Propagation Magazine, vol. 55, no. 3, pp. 217-223, 2013.

[10] M. Lott, I. Forkel, "A Multi-Wall-and-Floor Model for Indoor Radio Propagation", In IEEE $53^{\text {rd }}$ Vehicular Technology Conference, vol. 1, pp. 464-468, 2001

[11] Y. Akaiwa, "Introduction to Digital Mobile Communication - Second Edition", Chichester: John Wiley \& Sons, 2015.

[12] W. C. Y. Lee, "Mobile Cellular Telecommunications Systems", NewYork: McDraw-Hill, 1989.

[13] A. Chandra, A. Kumar, P. Chandra, "Estimation of Path Loss Parameters Using Propagation Measurements at $900 \mathrm{MHz}$ and $1.89 \mathrm{GHz}$ in The Corridors of a Multifloor Building”, IEEE Spread Spectrum Techniques and Applications, pp. 532-535, 1998 .

[14] B. Zhang, Z. Zhong, X. Zhou, K. Guan, R. He, "Path Loss Characteristics of Indoor Radio Channels at $15 \mathrm{GHz}$ ", $201610^{\text {th }}$ European Conference on Antennas and Propagation, pp. 1-5, 2016.

[15] J. Sadowski, J. Stefański, P. Rajchowski, K. Cwalina, P. Gilski, J. Magiera, "Remote Monitoring System of Persons Position in Indoor Environment (in Polish)", Telecommunication Review and Telecommunication News, no. 6, pp. 365-368, 2016.

[16] D. Tse, "Fundamentals of Wireless Communication", University of California, Berkeley: Cambridge University Press, 2005.

[17] Y. P. Zhang, "Novel Model for Propagation Loss Prediction in Tunnels", IEEE Transactions on Vehicular Technology, vol. 52, pp. 1308-1314, 2003.

[18] R. J. Katulski, A. Kiedrowski, "Calculation of the Propagation Loss in Urban Radio-Access Systems", IEEE Antennas and Propagation Magazine, vol. 50, pp. 65-70, 2008.

[19] Rohde \& Schwarz, "Vector Signal Generator - Specifications SMBV100A", Technical Data Sheet, 2010.

[20] Cobham Antenna Systems, "VHF/UHF Whip Antenna - OA1-0.030.50V/2013", Technical Data Sheet, 2013.

[21] Rohde \& Schwarz, "Active Directional Antenna - HE300", Technical Data Sheet, 2014.

[22] Anritsu, "Spectrum Master - User Guide - MS2721B", Technical Data Sheet, 2008.

[23] S. J. Ambroziak, "Measuring research on radio wave propagation normative requirements (in Polish)", Telecommunication Review and Telecommunication News, vol. 2-3, pp. 84-89, 2010.

[24] ITU-R P.522-5 “Calculation of Free-Space Attenuation”, 1994.

[25] R. Kattenbach, ,Statistical and Empirical Modelling”, Wireless Flexible Personalised Communications, New-York: John Wiley \& Sons, 2001. 